\title{
Turistas y Residentes en Destinos de Sol y Playa de Argentina
}

\author{
Juan Carlos Mantero ${ }^{1}$ \\ Bernarda Barbini ${ }^{2}$ \\ Marcela Bertoni ${ }^{3}$
}

\section{RESUMEN}

El presente trabajo aspira a dar a conocer resultados y reflexiones de una investigación empírica respecto de la sociabilidad del turista y de la relación turista residente en período de ocio vacacional en centros turísticos del litoral Atlántico Bonaerense, destinos de sol y playas.

La investigación en curso, Estudio de los centros turísticos del litoral, se aboca a la consideración de diferentes dimensiones de la problemática conformada por la actividad turística. A fin de considerar la dimensión socio - cultural se han realizado encuestas a turistas y residentes de estos centros que acogen una significativa afluencia turística de temporada.

Las encuestas realizadas a residentes y turistas de siete centros del litoral han permitido una caracterización de la identidad y diversidad en la conformación de la residencia y la afluencia de personas y la diferencial caracterización socioeconómica de los sujetos, las

\footnotetext{
1 Juan Carlos Mantero: Arquitecto. especializado en ordenación territorial y en gestión ambiental del desarrollo urbano. Profesor Titular de Política Turística y Recreativa y de Planificación del Turismo y la Recreación. Director del Centro de Investigaciones Turísticas. Director de revista APORTES y Transferencias. Tiempo Libre, Turismo y Recreación. Facultad de Ciencias Económicas y Sociales. Universidad Nacional de Mar del Plata. Argentina. e-mail jmantero@mdp.edu.ar

2 Bernarda Barbini: Licenciada en Sociología. Tesis en realización de Maestría en Ciencias Sociales de FLACSO. Docente de Sociología y de Sociología del Turismo e investigadora del Centro de Investigaciones Turísticas de la Facultad de Ciencias Económicas y Sociales de la Universidad Nacional de Mar del Plata. e-mail: bbarbini@mdp.edu.ar

3 Marcela Bertoni: Licenciada en Turismo. Tesis en realización Maestría de Gestión Ambiental del Desarrollo Urbano de la UNMDP. Docente de Teoría del Turismo y la Recreación y de Conservación de los Recursos e investigadora del Centro de Investigaciones Turísticas de la Facultad de Ciencias Económicas y Sociales de la Universidad Nacional de Mar del Plata. e-mail: marber@mdp.edu.ar
} 
expectativas y las prácticas de sociabilidad de los turistas y las recíprocas apreciaciones y ponderaciones generadas en la interacción turista-residente.

Los resultados permiten apreciar que, no obstante la unidad conferida por el recurso convocante y la relativa similitud de la procedencia, en el perfil del turista y del residente de cada centro se observan singularidades, producto de la conformación socio-económica de cada población considerada y en congruencia expectativas, prácticas y valoraciones diferenciales, que es menester considerar en la concepción de políticas turísticas y en la implementación de acciones de promoción y desarrollo.

\section{INTRODUCCIÓN.}

El Litoral Atlántico de la Provincia de Buenos Aires en Argentina se extiende 1.280 kms., desde el cabo San Antonio hasta la desembocadura del río Patagones, y comprende una serie de Municipios con un número elevado centros destinados a la actividad turística. No obstante, tal como se indica en la presentación el presente trabajo se remite a centros localizados en los Municipios de La Costa, Pinamar, Villa Gesell y Mar Chiquita, donde se ubican los centros objeto de investigación, afectados al turismo de sol y playas (Provincia de Buenos Aires. Centros: San Clemente, Santa Teresita, San Bernardo, Mar de Ajó, Pinamar, Villa Gesell, Santa Clara).

El modelo turístico de sol y playa de los centros turísticos del litoral Atlántico Bonaerense se ha desarrollado, desde hace cinco décadas, con el propósito de dar respuesta al turismo masivo inducido, promovido para una demanda interna limitadamente diferenciada, caracterizándose por una oferta de un producto estandarizado de considerable rigidez sustentado en el recurso natural: la playa.

Los destinos de sol y playa, comprendidos en los Municipios mencionados, reciben una afluencia masiva del turismo interno de Argentina, que supera los tres millones de turistas en temporada, de acuerdo a información de la Subsecretaria de Turismo de la Provincia de Buenos Aires.

Además de concentrada en el espacio de la franja litoral, la afluencia se concentra en el tiempo, la temporada, en un lapso que se extiende desde la segunda quincena de diciembre a la primer quincena de marzo, determinando una acentuada estacionalidad, considerable al promediar dicho período. 
La capacidad locacional de los Municipios donde se ubican los centros objeto de estudio es de aproximadamente 445.000 de plazas ${ }^{4}$, donde más de 40.000 son plazas en hotel, 400.000 plazas en vivienda, y las restantes plazas se distribuyen en otros tipos de alojamiento ${ }^{5}$. La rigidez de la oferta de alojamiento hace que en la totalidad de las localidades consideradas el tipo de alojamiento más utilizado sea el departamento o la casa, en propiedad y/o en alquiler.

El tipo de turismo es vacacional y familiar con fines de descanso y recreación sustentado en el recurso del mar y la playa, condición que supone cierta homogeneidad en las expectativas y las preferencias de los usuarios, permitiendo asumir que las variaciones en los niveles de uso se corresponden con las variaciones de su calidad.

Tales condiciones son determinantes de una modalidad turística que se caracteriza por la domesticidad de las prácticas turísticas (J. C. Mantero, 1997), atento a la incidencia que plantea un turismo en familia cuando coincide con un turismo en vivienda.

La domesticidad de las prácticas supone ciertas implicancias:

- La oferta de alojamiento se atomiza en unidades de vivienda de incierta equivalencia, multiplicando oferentes.

- El equipamiento de alojamiento no requiere prestación de servicios ad-hoc, en tanto la alimentación se realiza en la vivienda y la recreación tiene en la vivienda un ámbito alternativo.

- La capacidad de alojamiento es flexible (siempre cabe uno más) y el umbral de consumo es reducible (siempre se puede con algo menos), induciendo al turista a apelar a estrategias de subsistencia, de mínimo consumo.

Las tendencias señaladas en la modalidad turística suponen una actitud de repliegue del turista habitual y consecuente pérdida de dinámica en la inducción de actividades y servicios.

La reducción de consumos y servicios implica disminución del efecto multiplicador y de la generación de empleo atribuible a la actividad, en tanto la atomización en unidades de vivienda implica aumento de costo de funcionamiento y disuasión de tareas de acondicionamiento.

\footnotetext{
${ }^{4}$ Según datos del Sistema Integrado de Estadística Turística Provincial, Subsecretaria de Turismo de la Provincia de Buenos Aires.

${ }^{5}$ Las plazas hoteleras se distribuyen según la siguiente asignación: Municipio de la Costa 18.012, Municipio de Pinamar 11.466 y Municipio de Villa Gesell 13.270.

Respecto de las viviendas la distribución es 276.318 para La Costa, 52.385 para Pinamar y para Villa Gesell 74.556.

A propósito de los centros representativos, se consignan magnitudes de población residente al censo de 199 1: San Clemente 7.897 hab., Pinamar 6.075 hab., Villa Gesell 15.274 hab.
} 
El alcance de la domesticidad no subestima ciertas condiciones propicias, emergentes de la privacidad y la tranquilidad en el descanso aspirado, sino que advierte respecto de prácticas que requieren evaluación respecto de su incidencia en la actividad turística y el desarrollo urbano.

Por otra parte, este tipo de turismo ha generado y provocado impactos localizados espacial y temporalmente en las localidades, debido al patrón de crecimiento urbanístico expansivo, produciéndose una ocupación indiscriminada del suelo, asociada a la especulación inmobiliaria y la permisividad administrativa que ha implicado una política de hechos consumados.

Este crecimiento masivo no planificado ha producido inadecuación oferta/demanda, y degradación estética y funcional, que ponen de manifiesto signos de agotamiento y pérdida de competitividad y rentabilidad privada y social, aspectos que caracterizan la fase de estancamiento del producto; generando procesos de saturación en varios destinos.

El espacio turístico consolidado ha sido proyectado por y para el turismo y la inversión del sector privado esta directamente relacionada con la expectativa de la renta diferencial que garantizan ciertas condiciones paisajísticas y económicas de los espacios.

La competitividad de tales destinos turísticos en la mayor parte de los casos, presenta una prestación de servicios turísticos de limitada calidad, cuya variable de ajuste es el precio en la intención de incrementar el número de turistas.

La localización de servicios turísticos se concentra en el espacio adyacente a la playa, constituyendo una unidad funcional de espacio mercantilizado y saturado en el periodo estival, dispuesto para el consumo de equipamientos y espacios en su conjunto, que adquieren distinto significado para los actores sociales en función de su apropiación.

La disposición espacial presenta una fragmentación entre el área de servicios equipamientos turísticos y el área de residencia de la población local, que queda marginada de este espacio conformando una estructura territorial dispersa.

El desarrollo local de estos centros es muy dependiente de la propia evolución del sector turístico y de los diferentes grados de deterioro medio ambiental y paisajístico, de la masificación del espacio turístico, y de las dificultades de modernización de los equipamientos; factores que debilitan la posición de algunas localidades respecto de otras.

La situación consignada se acentúa si se considera que, a corto y mediano plazo, el sostenimiento de estos destinos turísticos esta relacionado con la oferta de sol y playa, y que el 
volumen de la demanda recurrente debe ser conservada garantizando su satisfacción creciente.

La satisfacción de la demanda supone la calidad del destino turístico a visitar, que implica actuaciones de conservación del recurso, la calificación de la prestación de servicios y la promoción de otros productos ejerzan un papel complementario y diversificador de los centros turísticos balnearios.

\section{ESTUDIO DE CENTROS TURISTICOS.}

\section{ENCUESTAS A TURISTAS Y RESIDENTES.}

La información objeto de análisis en el presente trabajo es resultado de encuestas realizadas en el contexto de la investigación en curso "Estudio de los Centros Turísticos del Litoral Atlántico Bonaerense", que comprende la indagación de las dimensiones socio-espacial, socio-cultural y socio-económica de la actividad turística en la modalidad de turismo de sol y playas.

Al abocarse a tales dimensiones, el estudio procura asumir las implicancias de la relación del habitante residente y del habitante estacional -los centros en tanto escenarios-, su relación con el ambiente -los centros en tanto soportes- y su relación con la producción y el consumo -los centros en tanto sustento-.

Así, la encuesta realizada a turistas permite no sólo conocer el perfil del turista sino disponer de información respecto de sus percepciones, proyecciones y valoraciones a propósito de recursos, servicios y condiciones de cada centro turístico y, en tal sentido, las preguntas resultan de un planteo de cuestiones y temas a propósito del turista: su perfil socioeconómico, expectativas y conductas, usos y consumos, grado de satisfacción, percepción y valoración con relación a actividades, servicios y centros.

La encuesta realizada a residentes incluye variables y categorías relacionadas con temas respecto de su problemática en centros turísticos de sol y playa, por tanto apropiados a la consideración genérica de asentamientos poblacionales urbanos y a la consideración específica de aquellos que además constituyen asentamientos poblacionales turísticos. Al margen de los aspectos que suelen ser objeto habitual de encuesta, tales como los que se refieren al perfil socio-demográfico del residente, se contemplan aspectos singulares como el nivel socioeconómico, el proceso de radicación, el trabajo y el tiempo libre y las percepciones y valoraciones sobre lo urbano y lo turístico. 


\section{TURISTAS Y RESIDENTES: IDENTIDAD Y DIVERSIDAD DEL NIVEL SOCIOECONOMICO.}

La conformación socioeconómica de turistas y residentes se define a través del análisis de indicadores referidos al capital económico y capital cultural, cuya apropiación diferencial, determina condiciones de existencia homogéneas que producen condicionamientos homogéneos, susceptibles de inducir prácticas turísticas semejantes.

La composición socioeconómica de tales conjuntos, no se determina únicamente por su posición en las relaciones producción material, es decir por la disposición de un capital económico; sino también por su ubicación en las relaciones de producción simbólica, dadas por la adquisición de capital cultural.

En tal sentido, la consideración de indicadores referidos a la apropiación de capital cultural, se considera primordial en el análisis de las expectativas de sociabilidad de los turistas y las apreciaciones y ponderaciones de los residentes en relación a la interacción con aquellos; ya que debe tenerse en cuenta la relación que une dichas expectativas y apreciaciones, con el capital educacional dado por el nivel de formación adquirido.

Por medio de las acciones de inculcación e imposición de valores que ejerce, la educación contribuye también a la constitución de disposiciones respecto de la cultura legítima, que tienden a aplicarse más allá de los límites de lo institucionalizado, creando una propensión a acumular determinadas experiencias y conocimientos. Así, las preferencias y percepciones de turistas y residentes, respecto de las formas de sociabilidad e interacción, serán producto de aprendizajes que hacen posible una disposición obtenida mediante la adquisición familiar y escolar de la cultura legítima. Provista de un conjunto de percepciones y de apreciaciones de aplicación general, esta disposición se transporta hacia todas las experiencias culturales, como es el caso del encuentro entre diferentes grupos provocado por la actividad turística; y permite percibirlas, clasificarlas y memorizarlas de distinta manera.

A partir de la consideración de estos indicadores, se definen grupos homogéneos con respecto a determinantes fundamentales de las condiciones materiales de existencia y de los condicionamientos que estas imponen, tomando en cuenta las redes de relaciones que se encuentran presentes en cada uno de estos factores.

El perfil socioeconómico de turistas y residentes se define a partir de una adaptación de la definición del índice de nivel socioeconómico adoptada por la Comisión de Investigación de la Asociación Argentina de Marketing, cuya formulación se considera adecuada a los fines del 
análisis planteado, ya que intenta definir grupos homogéneos contemplando la disposición diferencial, no solamente de capital económico, sino también cultural y simbólico, de uso apropiado en el contexto nacional.

En el caso de los turistas, se consideran y ponderan variables tales como nivel de educación, nivel de ocupación, posesión y tipo de automóvil, tipo y nivel de alojamiento turístico, destinos turísticos de últimas vacaciones realizadas, ponderando además su congruencia con el nivel de ingresos del grupo.

En el caso de los residentes, se contemplan y ponderan variables tales como nivel de educación, nivel de ocupación, posesión y tipo de automóvil, tipo, condición y nivel de vivienda ocupada, sin perjuicio de pondera congruencia con nivel de ingresos del grupo familiar.

En ambos grupos, la ponderación de estos indicadores permite la estratificación en cinco estratos: nivel alto, nivel medio-alto, nivel medio-medio, nivel medio-bajo, y nivel bajo.

Los indicadores adoptados, su ponderación relativa y la estratificación en función de umbrales apropiados, se consideran adecuados a los fines de la caracterización de los grupos turísticos y los grupos residentes; lo que permite definir su conformación socioeconómica de acuerdo al criterio adoptado.

Al considerar tal conformación, en relación al total de la muestra integrada por los turistas localizados en los diferentes centros, puede observarse que el nivel socioeconómico que presenta mayor proporción es el medio-medio y en orden decreciente los niveles medio bajo, medio-alto y bajo, observándose una homogeneidad genérica subyacente.

No obstante, al analizar las localidades, se distinguen tres centros representativos de diferentes perfiles socioeconómicos, así Pinamar es representativo del nivel medio-alto, Villa Gesell del nivel medio-medio y San Clemente del nivel medio-bajo.

En relación al total de la muestra integrada por los residentes de los centros turísticos considerados, se observa que los niveles socioeconómicos que se presentan en mayor proporción son, de acuerdo a su prelación, los niveles bajo y medio-bajo; consignándose proporciones significativamente menores en los niveles medio-medio y medio-alto; revelándose también una relativa homogeneidad.

Al comparar las localidades, cabe afirmar que si bien los niveles más representativos, en todos los centros, son el nivel bajo y el nivel medio-bajo, Villa Gesell se singulariza y se diferencia al cobrar relevancia el nivel medio-medio. 
Al observar la composición socioeconómica de la afluencia turística y de la población residente de los centros representativos mencionados, y no obstante el diferente rango observado en los niveles socioeconómicos de turistas y residentes considerados, se advierten proximidades y distancias entre dichos conjuntos, que definen tres estereotipos de correlación a partir de la congruencia / incongruencia observada en su confrontación.

Tales estereotipos resultan ser:

1. de congruencia de turistas y residentes en el nivel medio-medio en el caso de Villa Gesell,

2. de congruencia de turistas y residentes en el nivel medio-bajo en el caso de San Clemente y

3. de incongruencia de turistas de nivel medio-alto y de residentes de nivel medio-bajo en el caso de Pinamar

A partir de la definición de estos casos tipos de correlación turista - residente es factible inferir grados de permeabilidad, por tanto de predisposición y de apertura, en las formas de sociabilidad preferente de los turistas y en la interacción turista - residente que se acreditan en los respectivos análisis realizados.

\section{TURISTAS: LA SOCIABILIDAD PREFERENTE.}

Se entiende por formas de sociabilidad preferente a los tipos específicos de vínculos o relaciones que los turistas prefieren sostener y/o establecer en el ámbito de la práctica turística. Tales tipos específicos se definen a partir de la diferenciación de diversos modos de relación reconocibles que implican grados de afinidad y sugieren valorizaciones e intereses al respecto.

La relevancia del tipo de análisis ha de apreciarse al tener en cuenta que la sociabilidad es un aspecto importante del proceso de relación e integración, en tanto entran en juego tres tipos de factores: el entorno social, la participación dentro de grupos de referencia - pertenencia y las características individuales.

En tal consideración, el estudio de la socialización debe contemplar el tipo, la intensidad y la frecuencia, así como también subjetividad manifestada en la preferencia, de las interacciones que se establecen entre los individuos.

A fin de indagar sobre estas formas de sociabilidad de los turistas en los centros turísticos estudiados, se consideran las elecciones del turista respecto de la pregunta "Durante sus vacaciones Ud. Prefiere..." entre las alternativas de respuesta, estar con amigos - estar con gente de su nivel cultural - estar con gente de su lugar de procedencia - conocer gente de 
su nivel -conocer gente de diferentes niveles sociales - conocer gente residente de este centro turístico - compartir con grupo familiar de similar integración - compartir con gente de su edad compartir con gente de similar nivel socioeconómico.

Tal sistema de categorías contempla distintos tipos de motivaciones asociadas a la relación social que interesa mantener o generar y plantea distintas formas de establecerlos, considerando la disposición y la actividad diferenciales de conocer, estar, y compartir.

Las motivaciones se traducen en preferencias y pueden estar orientadas básica, aunque no exclusivamente, a las condiciones de amistad, de afinidad etárea o de similar conformación familiar, a la posesión de similar o de diferente capital social, cultural o económico y al lugar de residencia.

Al considerar estas formas de sociabilidad en la totalidad de la muestra integrada por los turistas, localizados en los diferentes centros, puede observarse que la forma de sociabilidad que concita más preferencias es estar con amigos y en orden decreciente, compartir con grupo familiar de similar integración y compartir con gente de su edad.

Al analizar lo que ocurre en cada localidad, puede constatarse que existen tendencias de homogeneidad genérica en las preferencias, orientadas a las condiciones de amistad, de estructura familiar similar y de estrato etáreo semejante, predominando las formas estar y compartir respecto de la de conocer.

En particular, al analizar la predisposición de los turistas a relacionarse con los residentes, en función de la opción conocer gente residente en este centro turístico, se observa una vinculación con los casos tipos establecidos de congruencia e incongruencia de los niveles socioeconómicos. Así, para el caso de congruencia turista / residente en el nivel medio-bajo se observa una notable permeabilidad, para el caso de congruencia turista/ residente en el nivel medio-medio se constata una permeabilidad intermedia y para el caso de incongruencia turista / residente se advierte una considerable impermeabilidad en la interacción.

En genérica consideración, a propósito de los diferentes centros considerados, podría afirmarse que los turistas no establecen interacciones informales con los residentes, en tanto no parecen procurar tal tipo de relación, sino más bien reproducir un entorno recreacional similar al realizado en su estilo de vida habitual.

El lugar de residencia de turistas y residentes es percibido y valorado como un atributo valorizante y desvalorizante, ya que los estilos de estos grupos dependerán de su posición en el espacio social, pero también de su distribución diferencial en un espacio geográfico, dado por la procedencia del turista y la localización del residente, que se encuentra socialmente 
jerarquizado, en función de su distribución -distancia o proximidad- respecto del centro de los valores económicos y culturales. (Bourdieu, 1999).

\section{RESIDENTES: LA INTERACCION TURISTA - RESIDENTE.}

En la interacción turista - residente surge una cultura del encuentro resultante de las formas adaptadas de visitantes y residentes que manifiestan diferencias respecto de las culturas matrices y donde cada una de ellas aporta, asimétricamente, parte de sus conceptos y valores, constituyendo una combinación cultural única.

La cultura del encuentro, integral o parcialmente, de modo gradual, debido a los impactos rutinarios del turismo, puede incorporarse a la forma original ordinaria de la cultura local, aún en el riesgo de homogeneizarse, en proceso de sustitución y/o solapamiento generando la problemática de la aculturación que, en el caso de conjuntos de similar contexto nacional, no resulta necesariamente conflictivo. (Santana, 1997)

En la relación turista - residente, el turista deviene un miembro circunstancial -en tiempo y espacio- de otra comunidad, generándose una especial relación entre el turista y el grupo social que le recibe; receptor que ve alterado su entorno cotidiano y necesita adaptarse a nuevas situaciones, a partir de recíprocos estereotipos definidos, donde se da un consumo diferencial de espacios con valoración social y se producen diferentes niveles de satisfacción de expectativas encontradas.

El encuentro está condicionado por restricciones temporales y espaciales, ya que el contacto para el turista se limita a la duración de la estadía y para el residente a la duración de la temporada; la concentración de la oferta de servicios turísticos y urbanos en el espacio litoral induce a la concentración de la demanda en espacios acotados, generando escenarios variados para los distintos actores.

Por ello, la interacción turista/residente se da en el marco de un proceso conformado por situaciones diferenciadas entre el turista distendido y el residente ocupado, eventualmente desocupado y por tanto excluido, que inciden en su integración, generando tensiones especialmente en los espacios de ocio.

Tal interacción implica la coexistencia de dos realidades separadas, el universo del turista y el universo del residente, que conviven en un espacio físico y social que les incluye y donde se presentan tres contextos básicos de encuentros: 
- Cuando el turista adquiere un bien o servicio del prestatario.

- Cuando el turista y el residente se encuentran uno junto al otro en lugares de ocio.

- Cuando las dos partes se encuentran cara a cara con objeto de intercambiar información e ideas que faciliten su entendimiento (De Kadt, 1979).

Con el objeto de indagar sobre las características de la relación-interacción en centros turísticos estudiados, se consideran los grados de acuerdo-desacuerdo expresados por los residentes en relación a ciertas proposiciones concebidas al efecto.

\section{El encuentro en lugares para la provisión de bienes y la prestación de servicios.}

Al analizar el grado de conformidad respecto de la proposición "al residente no le importa cuántos y cuáles turistas vienen en temporada" y remitirse al total de los residentes encuestados, se constata que las opciones consignadas, en orden de importancia de acuerdo a la significación atribuida, son en desacuerdo, ni de acuerdo/ni en desacuerdo y de acuerdo.

Al considerar el grado de conformidad respecto de la proposición "lo malo es que los turistas que vienen gastan poco (temporada) y remitirse al total de residentes los encuestados, se manifiesta que las opciones consignadas en orden de importancia de acuerdo a la significación atribuida son de acuerdo, ni de acuerdo/ni en desacuerdo y en desacuerdo.

Al observar lo que ocurre en cada localidad puede constatarse que existe una homogeneidad genérica respecto de las ponderaciones manifestadas sobre estas afirmaciones que es extensiva a los estereotipos de congruencia e incongruencia mencionados.

En tal sentido, los residentes manifiestan que les concierne e importa el número y el tipo de turistas que les visitan, acreditando ponderar la diferenciación de flujos turísticos y su conciencia respecto de la cantidad y calidad de sus integrantes.

Sin embargo, el interés de esta distinción se centra en la idea de que el turista es fundamentalmente una fuente de ingresos, advirtiéndose un proceso de despersonalización en la apreciación del otro, traduciéndose en una relación carente de espontaneidad que implica necesidad, funcionalidad y utilidad recíproca, teniendo como fin primordial y casi excluyente la transacción económica.

\section{El encuentro en los lugares de ocio.}

Al analizar el grado de conformidad respecto de la proposición "para el residente es difícil integrarse con los turistas en la diversión", se comprueba que las opciones consignadas 
de acuerdo a la significación atribuida son en desacuerdo, ni de acuerdo/ni en desacuerdo y de acuerdo, observándose cierta dispersión dada por la similitud en la distribución de las opciones.

La proporción similar de desacuerdo y acuerdo respecto de la proposición expresa una heterogeneidad relativa evidenciando que se presumen tensiones en el encuentro en los espacios de ocio.

Al considerar lo que ocurre en cada localidad, ateniéndose a los centros representativos de congruencia - incongruencia de niveles socioeconómicos del turista y del residente, se constata en el caso de congruencia en el nivel bajo cierta indiferencia respecto de la afirmación, en el caso de congruencia en el nivel medio-medio se advierte disposición a integrarse y en el caso de incongruencia de niveles se manifiesta la percepción de inviabilidad de la integración.

Cabe aclarar que la percepción y ocupación de los espacios de ocio por parte de los turistas y residentes se presentan en forma diferenciada, atento que su disfrute se limita a algunos segmentos de usuarios.

\section{El encuentro cotidiano.}

Al analizar el grado de conformidad respecto de la proposición "es imposible convivir con el turista como vecino" se constata que las opciones consignadas en prelación, de acuerdo a la significación atribuida, son en desacuerdo, ni de acuerdo/ni en desacuerdo y de acuerdo.

Al observar el grado de conformidad respecto de la proposición "la relación en la calle entre turistas y residentes es conflictiva" y remitirse al total de residentes encuestados, se verifica que las opciones consignadas en prelación son en desacuerdo, ni de acuerdo/ni en desacuerdo y de acuerdo.

La relación del residente con el turista como vecino parece acreditar una cierta distensión, la gradación respecto de los centros representativos identificados plantea que en el caso de congruencia en el nivel bajo, la relación es neutra, en el caso de congruencia en el nivel medio-medio, se insinúa cierto grado de tensión, en tanto en el caso de incongruencia entre niveles no se advierte tensión o conflicto, producto de una segregación espacial que en Pinamar excluye la relación de vecindad.

Respecto de la relación en la calle entre residentes y turistas, los resultados de la encuesta constatan neutralidad en el caso de congruencia en el nivel medio-bajo, distensión en el caso de congruencia en el medio-medio, infiriéndose cierto grado de tensión y conflictividad potencial en el caso de incongruencia entre niveles. 
En síntesis, a propósito de los tres contextos de encuentro considerados, esta relación, debido a las restricciones temporales y espaciales que influyen en la intensidad y duración del contacto, genera experiencias diversas y diferentes, asimétricas y desequilibradas, entre turistas y residentes.

Para los residentes la relación con los turistas tiene consecuencias cotidianas, lo que implica una tendencia a compatibilizar preferencias y prácticas, aceptar contradicciones en las relaciones sociales y procurar convivencia en su proyección espacial, consecuencia de la valorización del turismo como perspectiva económica y del turista como cliente y consumidor.

\section{CONCLUSIONES Y REFLEXIONES.}

Las características del turismo de sol y playas que se desarrolla en el litoral atlántico, cuyos centros turísticos y actores primordiales fueran objeto de investigación, plantean que dentro de la unidad conferida por los atributos genéricos de su calidad de partícipes de una sociedad nacional, es factible identificar ciertas singularidades del turista y del residente de cada centro.

A propósito de atributos, similares o diferenciales, conferidos por el nivel socioeconómico del turista y del residente, el modo de sociabilidad del turista con sus semejantes y el modo de interacción del residente con el turista, es dable advertir que roles, niveles, modos y relaciones generan disposiciones relativamente similares que, sin embargo, adquieren expresiones dispares en cada caso, en función de la distancia socio-económica y socio-cultural entre unos y otros y de la conformación socio-espacial del centro turístico, de allí la singularidad de los centros representativos identificados.

No obstante, estar excluidos los niveles socioeconómicos extremos del uso turístico de los centros del litoral, por exclusión voluntaria del nivel más alto y exclusión forzosa del nivel más bajo, se observan distancias diferenciales de nivel socioeconómico entre turistas y residentes de los centros considerados que remiten a los modos de sociabilidad de los turistas y a los modos de interacción con los residentes.

En los centros del litoral norte (San Clemente, Santa Teresita, San Bernardo y Mar de Ajó), de simultánea generación -décadas del 30 y 40- y similar conformación -fraccionamiento suburbano-, destino preferente de flujos -migrantes y turísticos- del área metropolitana sur de Buenos Aires, es observable que el perfil del turista se corresponde en primacía con el nivel socioeconómico medio-bajo en tanto y, en relativa congruencia, el perfil del residente se 
correlaciona con el nivel socioeconómico bajo, acreditando proximidad en los niveles comprendidos.

El modo de sociabilidad del turista reivindica la afinidad de la familia, la amistad y la edad y la disposición, por primacía del nivel medio-bajo, de conocer gente residente y de otros niveles sociales.

La percepción de la interacción turista - residente por parte del residente es de permeabilidad, en tanto no objeta ni critica la cotidianeidad en la calle, ni la relación de vecindad, ni la integración en la diversión, acreditando proximidad en los niveles socioeconómicos de pertenencia.

En los centros del litoral sur de la región considerada (Pinamar, Cariló y Villa Gesell), de generación más reciente -décadas del 60 y 70- y análoga conformación -forestación urbanizada- destino preferente de flujos de diferentes procedencias, en particular de Buenos Aires y norte del área metropolitana, es apreciable que el perfil del turista se corresponde en primacía con el nivel socioeconómico medio-medio y medio-alto, en tanto, en sensible diferencia, el perfil del residente se inscribe en el nivel socio económico bajo, excepto en el caso de Villa Gesell que se corresponde al nivel medio-bajo, en tanto migrante de expectativa diferente.

El modo de sociabilidad del turista reivindica la afinidad de la amistad y de la familia y se aprecia la ínfima disposición, en tanto primacía del nivel medio-medio y medio-alto de conocer gente residente y de otros niveles sociales.

La percepción del modo de interacción por parte del residente respecto del turista es diferente respecto de los centros del norte, en tanto allí donde se observa incongruencia de niveles y congruencia en el nivel medio-medio se aprecia tensión en la relación cotidiana de calle que la segregación funcional no hace perceptible en la diversión. En el caso de Villa Gesell, la relación turista/residente hace crisis en el ámbito de la vecindad a pesar de acreditar congruencia en el nivel de pertenencia, en función de una valorizada apreciación del residente respecto de las condiciones y requisitos de su residencialidad.

De los atributos consignados respecto de los actores, su nivel socioeconómico de referencia, sus modalidades de sociabilidad y sus expectativas de interacción, resultan singularidades que, sustentadas en las condiciones propias de las localidades, de su urbanización -escena y paisaje-, de su devenir turístico -residencial o productivo- y de su entidad local -identidad y pertenencia-, permiten diferenciar posibilidades y potencialidades de centros turísticos en búsqueda y proposición de opciones apropiadas a su estado y proyección. 
Atento la diferente incidencia que tiene la situación social en la diversificada afluencia turística, producto del ajuste de la economía, y el consecuente impacto en la población residente de cada uno de los centros, es menester concebir políticas adecuadas al estado y devenir de cada centro, en función del impacto recesivo en el turista recurrente y en el residente que habita.

La consideración de los centros turísticos del litoral como una unidad de limitadas variantes puede inducir a error y generar la adopción de políticas similares para atender y resolver situaciones dispares.

Al trascender las diferencias turísticas y urbanísticas -obviadas en la presente comunicación- y detenerse en la apreciación de perfil de los actores, en niveles referenciales de lo cultural, social y económico, acreditados en modalidades de sociabilidad del turista y de interacción del residente, puede inferirse la adopción de programas tendientes a capitalizar las modalidades dominantes -de sociabilidad e interacción- en la concepción de actividades y servicios que generen satisfacción del turista sin mediar tensión y/o in-satisfacción del residente, independientemente de las utilidades diferenciales a que aspira el uno y el otro, en orden a lo funcional, simbólico y vivencial.

La magnitud de la afluencia turística y de la población residente, la condición de la domesticidad de las prácticas -de turista en familia alojado en vivienda- y de la residencialidad de la actividad -de alojamiento y restauración en vivienda sin mediación de servicios turísticos-, la acentuada estacionalidad de la convocatoria, constituyen indicadores de una situación que tiende a asemejar la problemática de los diversos centros. No obstante, las diferencias de conformación urbana, por tanto la disposición y la aptitud de equipamientos y servicios, y de integración social, por tanto la capacidad y actitud de residentes y prestadores, permiten reconocer un umbral diferente para afrontar las dificultades y proponer las acciones apropiadas.

Al presente, las estrategias de promoción y desarrollo tienden a concebirse en función del turista actual y potencial con prescindencia de la consideración integrada del turista y del residente, que, según los niveles socioeconómicos de pertenencia y la distancia recíproca de niveles, modalidad de sociabilidad entre turistas y de interacción entre turistas y residentes, suponen sustento de acciones destinadas a promover y desarrollar un producto $\mathrm{o}$ un destino turístico cuando se trata de turistas del país en destinos vacacionales dentro del país.

La disposición resultante de la afinidad o disparidad entre niveles de los turistas y residentes, de las preferencias en los modos de conocer, estar y compartir entre turistas y de las expectativas y percepciones de la hospitalidad del residente, se constituyen en elementos sinérgicos persuasivos de una promoción persona a persona u opciones de asociatividad de 
unidades de emisión y recepción, que tratándose de flujos y destinos nacionales adquieren una potencialidad habitualmente subestimada frente a la seducción de los medios publicitarios y de comunicación social masiva, en el caso del turismo de sol y playas de índole vacacional en centros predominantemente residenciales acreditado en encuestas periódicamente realizadas.

La investigación en curso, contexto de las encuestas referidas en el presente trabajo, comprende la consideración pluridimensional del fenómeno turístico en una región afectada al turismo de sol y playas con un devenir urbano y poblacional que plantea un rol creciente del residente en la asunción de una problemática de promoción y desarrollo que excede lo turístico propiamente dicho y que supone el necesario reconocimiento de niveles socioeconómicos, necesidades y aspiraciones, posibilidades y restricciones, de la sociedad local para afrontar las vulnerabilidades de una actividad estacionalizada y sensible a las condiciones de contexto nacional, que en su incidencia afecta tanto al turista como al residente.

La aptitud de los diferentes centros para afrontar problemas de cierta recurrencia radica en las condiciones de sustento del territorio natural, relativamente similar, y del territorio antropizado, sensiblemente diferente por oportunidad y modo de generación del centro (fraccionamientos urbanos en loteos especulativos de las décadas del 40/50 -centros del norte de la región- y urbanizaciones turísticas en unidades forestadas de las décadas del 60/70 centros del sur de la región-) y, primordialmente en las condiciones y disposiciones de la población residente y las aspiraciones y exigencias de la afluencia turística.

Conocer y reconocer las condiciones culturales, sociales y económicas de la población residente y afluencia turística, sus modos de relación e interacción, parcialmente considerados en la presente comunicación, permiten advertir ámbitos de acción tendientes a atenuar vulnerabilidades e identificar oportunidades que trasciendan la actividad turística y permitan asumir la problemática de territorios que nacidos fraccionamientos devienen urbanizaciones turísticas y, en ciertos casos, por la magnitud y actitud de sus residentes y migrantes se constituyen en centros urbanos que aspiran a superar las restricciones de un turismo doméstico y estacional y las fragilidades propias del monocultivo de su producción de bienes y servicios. 
BIBLIOGRAFIA.

- Agenda 11 Seminario estrategias para el desarrollo turístico: Producto Playa, Villa Gesell, 1993.

- Boullon, Roberto: Los Municipios Turísticos. Ed. Trillas Méjico, 1990.

- Bourdieu, Pierre: La distinción, Ed. Taurus, Madrid, 1999.

- Cicalesse, Guillermo: Administración y apropiación del recurso playa en una fase de crisis del turismo: Mar del Plata, 1983-1993. Cuadernos de Estudios Turísticos N 1.

- Court, Birgit y Lupton Robert: Custumer Portfolio Development: Modeling Destination, Adopters, Inactives, Rejecters. Journal of Travel Research, Volume XXXVI, NQ 1, Verano 1997.

- De Kadt, Emanuel: Turismo: ¿Pasaporte al desarrollo? Ediciones Endyrnion, 1979.

- Donaire, José y otros: La costa Brava ante los nuevos retos del turismo, Estudios Turísticos, N2 133, 1997, pp.77-96.

- Grunewald, Luis: La competitividad del producto "sol y playa". Diagnóstico de las fortalezas y debilidades del producto "Sol y Playa" en el corredor de las Playas Bonaerenses, en Plan de competitividad de la oferta de alojamiento de Villa Gesell, Cámara de Empresarios Hoteleros, 1997.

- Mantero, Juan Carlos y Cañueto Jorge: Miramar: Dimensiones críticas y estrategias de desarrollo. Aportes y Transferencias, Año 1 Volumen 1, mayo de 1997.

- Mantero, Juan Carlos y Otros Mar del Plata: Afrontar la estacionalidad, en Aportes y Transferencias, Año 1 Voll, 1997.

- Mantero, Juan Carlos, Barbini, Bernarda y Bertoni, Marcela: Encuesta a Residentes en Centros Turísticos del Litoral Atlántico, en Aportes y Transferencias, Año 3 Volumen 1, abril de 1999.

- Saiz Mujica, Ignacio: Turismo, medio ambiente y ordenación del territorio en los espacios litorales, en España es un país turísticamente avanzado, AICET Instituto de Estudios Turísticos, Marbella, 1994.

- Santana, Agustín: Antropología y Turismo, ¿Nuevas hordas, viejas culturas?, Ed. Ariel, SA. España, 1997.

- Vera Rebollo, Fernando y Monforte Mir, Vicente: Agotamiento de los modelos turísticos clásicos. Una estrategia territorial para la cualificación: La experiencia de la Comunidad Valeneciana, en Estudios Turísticos, W 123, pp. 17-45, 1994. 